\title{
Homotopy Perturbation Method for Solving Linear Fuzzy Delay Differential Equations Using Double Parametric Approach
}

\author{
Ali F Jameel ${ }^{1, *}$, Sardar G Amen ${ }^{1,2}$, Azizan Saaban ${ }^{1}$, Noraziah H Man ${ }^{1}$, Fathilah M Alipiah ${ }^{1}$ \\ ${ }^{1}$ School of Quantitative Sciences, College of Art and Sciences, Universiti Utara Malaysia (UUM), Malaysia \\ ${ }^{2}$ Department of Financial and Banking, College of Business Administration and Financial Science, Al-Kitab University, Iraq
}

Received April 13, 2020; Revised July 6, 2020; Accepted July 20, 2020

(a): [1] Ali F Jameel, Sardar G Amen, Azizan Saaban, Noraziah H Man, Fathilah M Alipiah, "Homotopy Perturbation Method for Solving Linear Fuzzy Delay Differential Equations Using Double Parametric Approach," Mathematics and Statistics, Vol. 8, No. 5, pp. 551 - 558, 2020. DOI: 10.13189/ms.2020.080508.

(b): Ali F Jameel, Sardar G Amen, Azizan Saaban, Noraziah H Man, Fathilah M Alipiah (2020). Homotopy Perturbation Method for Solving Linear Fuzzy Delay Differential Equations Using Double Parametric Approach. Mathematics and Statistics, 8(5), 551 - 558. DOI: 10.13189/ms.2020.080508.

Copyright $(2020$ by authors, all rights reserved. Authors agree that this article remains permanently open access under the terms of the Creative Commons Attribution License 4.0 International License

\begin{abstract}
Delay differential equations (known as DDEs) are a broad use of many scientific researches and engineering applications. They come because the pace of the shift in their mathematical models relies all the basis not just on their present condition, but also on a certain past cases. In this work, we propose an algorithm of the approximate method to solve linear fuzzy delay differential equations using the Homotopy Perturbation Method with double parametric form fuzzy numbers. The detailed algorithm of the approach to fuzzification and defuzzificationis analysis is provided. In the initial conditions of the proposed problem there are uncertainties with regard to the triangular fuzzy number. A double parametric form of fuzzy numbers is defined and applied for the first time in this topic for the present analysis. This method's simplicity and ability to overcome delay differential equations without complicating Adomian polynomials or incorrect nonlinear assumptions. The approximate solution is compared with the exact solution to confirm the validity and efficiency of the method to handle linear fuzzy delay differential equation. To show the features of this proposed method, a numerical example is illustrated, involving first order fuzzy delay differential equation. These findings indicate that the suggested approach is very successful and simple to implement.
\end{abstract}

Keywords Fuzzy Delay Differential Equations (FDDE), Homotopy Perturbation Method (HPM),
Approximate Methods, Single Parametric form Fuzzy Numbers, Double Parametric form Fuzzy Numbers

\section{Introduction}

The variety of life experiments in science and engineering can be formulated in the form of ordinary or partial differential equation models. Models that involve delaying differential equations (DDEs) are defined as type of differential equations where the time derivatives at the current time depend on the solution and possibly its derivatives at previous times. A class of such equations, which involve derivatives with delays as well as the solution itself, has been called neutral DDEs [1,2]. For instance, drives, sensors and field networks involved in feedback loops can involve delays. In epidemic dynamics, time delay system is also used for designing multiple different mechanisms [3]. Most DDEs that arise in population dynamics and epidemiology model intrinsically have nonnegative quantities. Therefore, it is important to establish that nonnegative initial data which give rise to nonnegative solutions.

For modeling a dynamic system where the information regarding its behavior is insufficient, Fuzzy differential equations (FDEs) are known as a useful tool [4]. When 
these experiments are not modelled completely and their nature is uncertain or vague, fuzzy models can be used. FDEs are suited to modeling such dynamical systems that have been applied in many applications such as in population modelling [5,6], mathematical physics [7] and medical sciences [8]. Approximate methods were generally used to solve fuzzy delay differential equations (FDDEs) with single parametric form fuzzy numbers $[9,10]$.

Many researchers in the field of Science and Engineering have used Homotopy Perturbation Method (HPM) to achieve an approximate solution for different kinds of linear and nonlinear models [10-13], even the fuzzy partial differential equation models as in Sarmad et al [14]. Solving problems with HPM often help to better understand a physical problem, and may help improve future procedures and designs used to solve these problems. Also, this method has a useful feature in that it provides the solution in a rapid convergent power series with the elegantly computable convergence of the solution without any need for discretization and linearization as in numerical methods [15]. However, in using a single parametric form for HPM, a $\mathrm{n} \mathrm{n}$ fully fuzzy system has to be converted to $2 \mathrm{n} \times 2 \mathrm{n}$ crisp system. On the other hand, for the double parametric form, the $\mathrm{n} \times \mathrm{n}$ fully fuzzy system is converted to the same order of crisp system, hence requiring a less amount of computation. The double parametric form, which has been employed in fuzzy differential equation, is more general and straightforward [16].

Our aim here is to construct a new form of HPM based on the approach of double parametric form of fuzzy numbers to solve first order DDEs using fuzzy-set theory properties.

The outline of this research is as follows: Section 2 presents some tools and definitions of the fuzzy number for the fuzzy analysis of the fuzzy model. Section 3 introduces the defuzzification of the general FDDE in new fuzzy number form. In the Section 4, we modified the standard HPM into double parametric form fuzzy numbers form. Section 5, we implement the HPM in the Section 4 on first order linear FDDE to show the capability of the method. Finally section 6 presents the conclusions of this research.

\section{Fuzzy Numbers}

\subsection{Triangular Fuzzy Number [17]}

Fuzzy numbers are a subset of the real numbers set, and represent uncertain values. Fuzzy numbers are linked to degrees of membership referring to how true it is to say if something belongs or does not belong to a determined set. A fuzzy number $\mu$ is called a triangular fuzzy number if it is defined by three numbers $\alpha<\beta<\gamma$ where the graph of $\mu(x)$ is a triangle with the base on the interval $[\alpha, \beta]$ and vertex at $x=\beta$ as illustrated in Figure 1 and where its membership function is given by the following membership function $\mu(x ; \alpha, \beta, \gamma)$ :

$$
\left\{\begin{array}{l}
0, \quad \text { if } x<\alpha \\
\frac{x-\alpha}{\beta-\alpha}, \text { if } \alpha \leq x \leq \beta \\
\frac{\gamma-x}{\gamma-\beta}, \text { if } \beta \leq x \leq y \\
0, \quad \text { if } x>\gamma
\end{array}\right.
$$

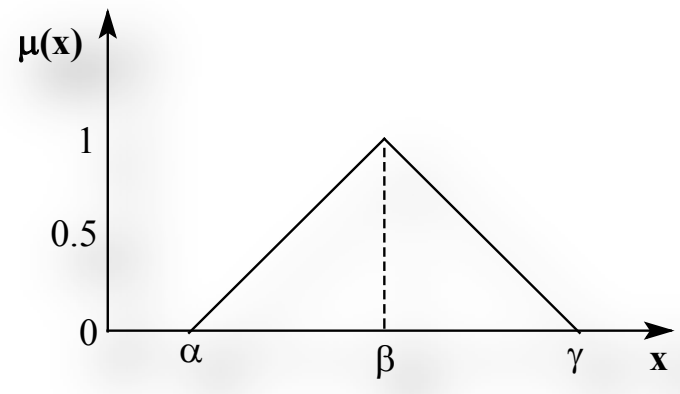

Figure 1. Triangular Fuzzy Number

and its $\quad r$-level is: $[\mu]_{r}=[\alpha+r(\beta-\alpha), \gamma-$ $r(\gamma-\beta)]$, for $r \in[0,1]$, where the $r$-level (or $r$-cut) set of a fuzzy set $\tilde{\mu}$, labeled as $\tilde{\mu}_{r}$, is the crisp set of all $x \in T \quad$ such that $\tilde{\mu}_{r} \geq r$, that is, $\tilde{\mu}_{r}=\left\{x \in T \mid \tilde{\mu}_{r}>r, r \in[0,1]\right\}[18]$.

Since the $r$-level set is the link between the fuzzy domain and the crisp domain, we can use the advantages of the theories in crisp domain and in the fuzzy domain such that $\frac{x-\alpha}{\beta-\alpha}=r \rightarrow r(\beta-\alpha)=x-\alpha \rightarrow x=\alpha+r(\beta-\alpha) \quad$ the lower bound of fuzzy number, and $\frac{\gamma-x}{\gamma-\beta}=r \rightarrow r(\gamma-\beta)=$ $\gamma-x \rightarrow x=\gamma-r(\gamma-\beta)$ upper bound of fuzzy number.

\subsection{Single Parametric form of Fuzzy Number [19]}

The class of all fuzzy subsets of $\mathbb{R}$ is denoted by $\tilde{E}$ and the solution of Fuzzy Initial Value Problem (FIVP) satisfy the following properties:

1. $\mu(x)$ is normal, that is, $\exists x_{0} \in \mathbb{R}$ with $\mu\left(x_{0}\right)=1$,

2. $\mu(x)$ is convex fuzzy set, that is, $\mu(\lambda x+(1-\lambda) t) \geq$ $\min \{\mu(x), \mu(t)\} \quad \forall x, t \in \mathbb{R}, \lambda[0,1]$,

3. $\mu(x)$ is upper semi-continuous on $\mathbb{R}$,

4. $\{x \in \mathbb{R}: \mu(x)>0\}$ is compact.

$\tilde{E}$ is called the space of fuzzy numbers and $\mathbb{R}$ is a proper subset of $\tilde{E}$.

Define the $r$-level set $x \in \mathbb{R},[\mu]_{r}=\{x \backslash \mu(x) \geq r\}, 0 \leq$ $r \leq 1$, where $[\mu]_{0}=\{x \backslash \mu(x)>0\}$ is compact, which is a closed bounded interval and denoted by $[\mu]_{r}=$ $[\underline{\mu}(x), \bar{\mu}(x)]$. In the single parametric form, a fuzzy number is represented by an ordered pair of functions $[\underline{\mu}(x), \bar{\mu}(x)], r \in[0,1]$ which satisfies:

1. $\mu(x)$ is a bounded left continuous non-decreasing function over $[0,1]$. 
2. $\bar{\mu}(x)$ is a bounded right continuous non-increasing function over $[0,1]$.

3. $\mu(x) \leq \bar{\mu}(x), r \in[0,1]$, where a crisp number $r$ is simply represented by $\mu(r)=\bar{\mu}(r)=r, r \in[0,1]$.

\subsection{Double Parametric Form of Fuzzy Number [20]}

Using the parametric form as defined in definition $\tilde{E}=$ $[\underline{\mu}(r), \bar{\mu}(r)]$, then, one may represent this in crisp number using double parametric form as $\tilde{E}(r ; \beta)=\beta[\bar{\mu}(r)-$ $\underline{\mu}(r)]+\underline{\mu}(r)$, where $r$ and $\beta \in[0,1]$. The parameter $\beta$ denotes the deform parameter such that if $\beta=0$ then $\tilde{E}(r ; 0)=\mu(r)$ (lower bound fuzzy number) and if $\beta=1$ then $\tilde{E}(r ; 1)=\bar{\mu}(r)$ (upper fuzzy number). In this way, the double parametric form provides less computational work than single parametric form.

\section{Fuzzy Delay Differential Equation in Double Parametric Form}

Consider the FDDEs [9].

$$
\begin{gathered}
\tilde{y}^{\prime}(x)=\tilde{f}(x, \tilde{y}(x), \tilde{y}(x-\alpha)), x \in\left[x_{0}, X\right] \\
\tilde{y}\left(x_{0}\right)=\tilde{y}_{0}
\end{gathered}
$$

where for all fuzzy level sets $r \in[0,1]$ and $\beta \in[0,1]$, we have the following defuzzifications:

1. The fuzzy functions $\tilde{y}(x)$ [19] is denoted as $[\tilde{y}(x ; r)]_{\beta}=\beta\left[\bar{y}\left(x_{0} ; r\right)-\underline{y}\left(x_{0} ; r\right)\right]+\underline{y}\left(x_{0} ; r\right)$,

2. The fuzzy delay functions $\tilde{y}(x-\alpha)$ is denoted as $[\tilde{y}(x-\alpha ; r)]_{\beta}=\beta[\bar{y}(x-\alpha ; r)-\underline{y}(x-\alpha ; r)]+$ $\underline{y}(x-\alpha ; r)$,

3. The fuzzy first order H-derivative (see [21])

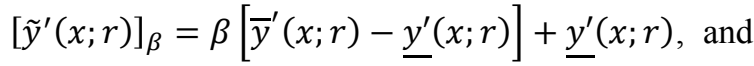
the fuzzy initial condition $\left[\tilde{y}\left(x_{0} ; r\right)\right]_{\beta}=\beta\left[\bar{y}\left(x_{0} ; r\right)-\underline{y}\left(x_{0} ; r\right)\right]+\underline{y}\left(x_{0} ; r\right)$ in the form of fuzzy triangular fuzzy number.

4. Let the fuzzy function $\tilde{f}(x, \tilde{y}(x), \tilde{y}(x-\alpha))=$ $\tilde{f}(x, \widetilde{U}(x))$ such that

$$
\tilde{f}(t, \widetilde{U}(x))=[\underline{f}(t, \widetilde{U}(x)), \underline{f}(t, \widetilde{U}(x))] .
$$

By using Zadeh extension principles [22], we have the following membership functions

$$
\begin{aligned}
& F(x, \widetilde{U}(x ; r))=\min \left\{\tilde{y}^{\prime}(x, \tilde{\mu}(r)): \mu \mid \mu \in[\widetilde{U}(x)]_{r}\right\}, \\
& G(x, \widetilde{U}(x ; r))=\max \left\{\tilde{y}^{\prime}(x, \tilde{\mu}(r)): \mu \mid \mu \in[\widetilde{U}(x)]_{r}\right\},
\end{aligned}
$$

Where

$$
\left\{\begin{array}{l}
\underline{f}(x, \widetilde{U}(x ; r))=F(t, \underline{U}(x ; r), \bar{U}(x ; r))=F(x, \widetilde{U}(x ; r)), \\
\bar{f}(x, \widetilde{U}(x ; r))=G(t, \underline{U}(x ; r), \bar{U}(t ; r))=G(x, \widetilde{U}(x ; r)) .
\end{array}\right.
$$

According to [23] and for all $r \in[0,1]$ and $\beta \in[0,1]$ the double parametric form is given in one equation as follows:

$$
\begin{gathered}
\beta\left[\bar{y}^{\prime}(x ; r)-\underline{y^{\prime}}(x ; r)\right]+\underline{y^{\prime}}(x ; r)=\beta[G-F]+F, \\
\beta\left[\bar{y}\left(x_{0} ; r\right)-\underline{y}\left(x_{0} ; r\right)\right]+\underline{y}\left(x_{0} ; r\right) \\
=\beta\left[\overline{y_{0}}(r)-\underline{y_{0}}(r)\right]+\underline{y_{0}}(r) .
\end{gathered}
$$

\section{Fuzzy HPM in Double Parametric Form}

This section presents the analysis of HPM for approximately solving first order FDDE as in Eq. (1) under double parametric form fuzzy number. According to the fuzzy analysis by Ali et al in [24], to obtain the approximate solution of the first order fuzzy initial value problem by HPM we rewrite Eq. (3) as follows:

$$
\beta[L(\bar{y}(x ; r)-\underline{y}(x ; r))]+L \underline{y}(x ; r)=\beta[G-F]+F .
$$

$$
\begin{array}{r}
\beta\left[\bar{y}\left(x_{0} ; r\right)-\underline{y}\left(x_{0} ; r\right)\right]+\underline{y}\left(x_{0} ; r\right) \\
=\beta\left[\overline{y_{0}}(r)-\underline{y_{0}}(r)\right]+\underline{y_{0}}(r),
\end{array}
$$

where $L$ is the linear operators referring to the first order H-derivative of Eq.(4) such that $L=\frac{d}{d x}$ and $F, G$ are nonlinear operators followed by the inverse operators $L^{-1}=\int_{0}^{x} \widetilde{[.]}_{r} d \tau$. According to HPM, we construct the following homotopy equation:

$$
\begin{gathered}
H(x, p ; r ; \beta)=L[\beta(\bar{y}(x ; r)-\underline{y}(x ; r))+\underline{y}(x ; r)- \\
\left.\beta\left(\bar{y}_{0}(x ; r)-\underline{y}_{0}(x ; r)\right)-\underline{y}_{0}(x ; r)\right]+p\left[L \beta \left(\bar{y}_{0}(x ; r)-\right.\right. \\
\left.\left.\underline{y}_{0}(x ; r)\right)-\underline{y}_{0}(x ; r)-\beta[G-F]-F\right]=0
\end{gathered}
$$

where $p \in[0,1]$ is an embedding parameter to deform Eq. (5) and initial guessing functions can be defined as follows:

$$
\begin{gathered}
{\left[\tilde{y}_{0}(x ; r)\right]_{\beta}=\beta\left(\bar{y}_{0}(x ; r)-\underline{y_{0}}(x ; r)\right)-\underline{y_{0}}(x ; r)=} \\
\beta\left[\overline{y_{0}}(r)-\underline{y_{0}}(r)\right]+\underline{y_{0}}(r) .
\end{gathered}
$$

For all $x \in\left[x_{0}, X\right]$ and from Eqs. (5-6) we have

$$
\left\{\begin{array}{c}
H(x, 0 ; r ; \beta)=L\left[\begin{array}{c}
\beta(\bar{y}(x ; r)-\underline{y}(x ; r)) \\
+\underline{y}(x ; r) \\
-\beta\left(\bar{y}_{0}(x ; r)-\underline{y}_{0}(x ; r)\right) \\
-\underline{y}_{0}(x ; r)
\end{array}\right]=0 \\
H(x, 1 ; r ; \beta)=L \beta\left(\bar{y}_{0}(x ; r)-\underline{y}_{0}(x ; r)\right) \\
-\underline{y}_{0}(x ; r)-\beta[G-F]-F=0,
\end{array}\right.
$$


where $p$ changes from 0 to 1 . Eqs. (6-7) are called deformation homotopy. The usage of $p$ as a small parameter is to construct HPM series function from Eq. (7) such that each $\tilde{y}(t ; r)$ in Eq. (3) follows

$$
\tilde{y}(x ; r ; \beta)=\sum_{k=0}^{\infty} p^{k} \tilde{y}_{k}(x ; r ; \beta)
$$

Finally, substitute Eq. (8) in Eq. (5) and then collect all terms of the same powers of $p$ such that $r \in[0,1]$ and $\beta \in[0,1]$ such that:

$$
\begin{gathered}
p^{0}:\left\{\begin{array}{c}
\beta\left(\bar{y}_{0}(x ; r)-\underline{y_{0}}(x ; r)\right)+\underline{y}_{0}(x ; r) \\
=\beta\left[\overline{y_{0}}(r)-\underline{y_{0}}(r)\right]+\underline{y_{0}}(r),
\end{array}\right. \\
p^{1}:\left\{\begin{array}{c}
{\left[\begin{array}{c}
\left.\beta\left(\bar{y}_{1}(x ; r)-\underline{y}_{1}(x ; r)\right)+\underline{y}_{1}(x ; r)\right] \\
+\beta\left(\bar{y}_{0}(x ; r)-\underline{y}_{0}(x ; r)\right)+\underline{y}_{0}(x ; r)
\end{array}\right]-} \\
\beta\left[G_{0}-F_{0}\right]-F_{0}=0
\end{array}\right] \\
p^{2}:\left\{\begin{array}{c}
L\left[\bar{y}_{1}\left(x_{0} ; r\right)-\bar{y}_{1}\left(x_{0} ; r\right)\right]+\bar{y}_{1}\left(x_{0} ; r\right)=0, \\
\left.\beta\left(\bar{y}_{1}(x ; r)-\underline{y}_{1}(x ; r)\right)+\underline{y}_{1}(x ; r)\right] \\
\beta\left[\bar{y}_{2}\left(x_{0} ; r\right)-\bar{y}_{2}\left(x_{0} ; r\right)\right]+\bar{y}_{2}\left(x_{0} ; r\right)=0,
\end{array}\right. \\
p^{k+1}:\left\{\begin{array}{c}
L\left[\begin{array}{c} 
\\
\beta
\end{array}\left(\bar{y}_{k}(x ; r)-\underline{y}_{k}(x ; r)\right)+\underline{y}_{k}(x ; r)\right] \\
-\beta\left[G_{k}-F_{k}\right]-F_{k}=0 \\
\beta\left[\bar{y}_{k}\left(x_{0} ; r\right)-\bar{y}_{k}\left(x_{0} ; r\right)\right]+\bar{y}_{k}\left(x_{0} ; r\right)=0 .
\end{array}\right.
\end{gathered}
$$

Then the approximate solution is given by setting $p=1$ as follows:

$$
\begin{gathered}
\beta(\bar{y}(x ; r)-\underline{y}(x ; r))+\underline{y}(x ; r)=\sum_{i=0}^{m-1} \beta\left(\bar{y}_{i}(x ; r)-\right. \\
\left.\underline{y_{i}}(x ; r)\right)+\underline{y}_{i}(x ; r) .
\end{gathered}
$$

In the standard HPM for solving fuzzy differential equation with fuzzy numbers with single parametric form, it is required to analyze the HPM for the lower and upper bound solution of Eq.(1) and hence would demand more computational work. The advantage of the parameter $\beta$ that deforms from 0 to 1 reduces the computational and analysis work to obtain the solution of fuzzy differential equations for illustration, at $\beta=0$ in Eq. (10) we obtain the lower solution of Eq. (1)

$$
\bar{y}(x ; r)=\sum_{i=0}^{m-1} \underline{y}_{i}(x ; r) .
$$

On the other hand at $\beta=1$ we obtain the upper solution of Eq. (1)

$$
\bar{y}(x ; r)=\sum_{i=0}^{m-1} \bar{y}_{i}(x ; r),
$$

and this is applicable for all the HPM analysis in double parametric form fuzzy numbers. Details and illustration are provided in the next section.

\section{Results and Discussion}

In this section, we present a numerical problem involving FDDE with an approximate solution obtained by HPM described in Section 4. This is followed by fuzzy analysis under double parametric form fuzzy number. Consider the linear FDDE with fuzzy initial conditions [9]:

$$
\tilde{y}^{\prime}(x)=\frac{1}{2} e^{\frac{x}{2}} \tilde{y}\left(\frac{x}{2}\right)+\frac{1}{2} \tilde{y}(x), \quad x \in[0,1],
$$

$$
\tilde{y}(0)=[r, 2-r], r \in[0,1],
$$

Then double parametric form of Eq. (13) is given by:

$$
\begin{gathered}
\beta\left[\overline{y^{\prime}}(x ; r)-\underline{y^{\prime}}(x ; r)\right]+y^{\prime}(x ; r)=\left\{\frac { 1 } { 2 } e ^ { \frac { x } { 2 } } \beta \left[\bar{y}\left(\frac{x}{2} ; r\right)-\right.\right. \\
\left.\left.\underline{y}\left(\frac{x}{2} ; r\right)\right]+\underline{y}\left(\frac{x}{2} ; r\right)+\frac{1}{2} \beta[\bar{y}(x ; r)-\underline{y}(x ; r)]+\underline{y}(x ; r)\right\}, \\
\beta[\bar{y}(0 ; r)-\underline{y}(0 ; r)]+\underline{y}(0 ; r)=\beta[2-2 r]+r,
\end{gathered}
$$

where $\beta \in[0,1]$ is a free parameter. According to [8] the exact solution of Eq. (13) in double parametric form is given by:

$$
\beta[2-2 r] e^{t}+r e^{t},
$$

If $\beta=0$ the exact lower solution in single parametric form is given by $\underline{y}(x ; r)=r e^{t}$, and if $\beta=1$ the exact upper solution in single parametric form is given by $\bar{y}(x ; r)=(2-r) e^{t}$.

$p^{0}:\left\{\beta\left(\bar{y}_{0}(x ; r)-\underline{y}_{0}(x ; r)\right)+\underline{y}_{0}(x ; r)=\beta[2-2 r]+r\right.$

$$
p^{1}:\left\{\begin{array}{c}
\beta\left(\bar{y}_{1}(x ; r)-\underline{y}_{1}(x ; r)\right)+\underline{y}_{1}(x ; r)= \\
L^{-1}\left[\begin{array}{c}
\frac{1}{2} e^{\frac{t}{2}} \beta\left[\bar{y}_{0}\left(\frac{t}{2} ; r\right)-\underline{y}_{0}\left(\frac{t}{2} ; r\right)\right]+\underline{y}_{0}\left(\frac{t}{2} ; r\right)+ \\
\frac{1}{2} \beta\left[\bar{y}_{0}(t ; r)-\underline{y}_{0}(t ; r)\right]+\underline{y}_{0}(t ; r) \\
\beta\left[\bar{y}_{1}\left(x_{0} ; r\right)-\bar{y}_{1}\left(x_{0} ; r\right)\right]+\bar{y}_{1}\left(x_{0} ; r\right)=0
\end{array}\right]
\end{array}\right.
$$

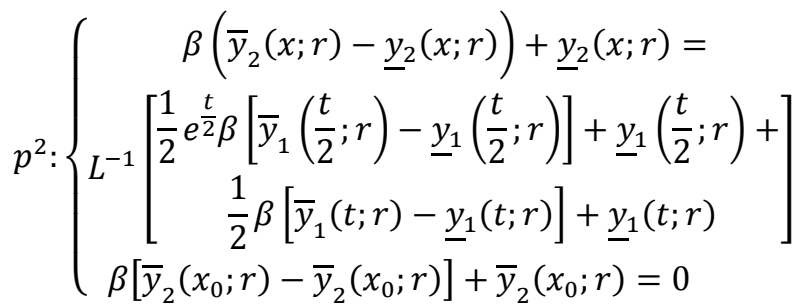

$$
\begin{aligned}
& p^{k+1}:\left\{\begin{array}{c}
\beta\left(\bar{y}_{k+1}(x ; r)-\underline{y}_{k+1}(x ; r)\right)+\underline{y}_{k+1}(x ; r)= \\
L^{-1}\left[\begin{array}{c}
\frac{1}{2} e^{\frac{t}{2}} \beta\left[\bar{y}_{k}\left(\frac{t}{2} ; r\right)-\underline{y}_{k}\left(\frac{t}{2} ; r\right)\right] \\
+\underline{y}_{k}\left(\frac{t}{2} ; r\right)+ \\
\frac{1}{2} \beta\left[\bar{y}_{k}(t ; r)-\underline{y}_{k 1}(t ; r)\right]+\underline{y}_{k}(t ; r)
\end{array}\right] \\
\beta\left[\bar{y}_{k+1}\left(x_{0} ; r\right)-\bar{y}_{k+1}\left(x_{0} ; r\right)\right]+\bar{y}_{k+1}\left(x_{0} ; r\right)=0
\end{array}\right.
\end{aligned}
$$


The HPM approximate solution series is given by:

$\tilde{y}(x ; r)=\sum_{i=0}^{5} \sum_{i=0}^{m-1} \beta\left(\bar{y}_{i}(x ; r)-\underline{y}_{i}(x ; r)\right)+\underline{y}_{i}(x ; r)$

at $x=1$. The absolute errors between fifth-order HPM and the exact solution for all $r \in[0,1]$ is denoted by

$$
[E(r ; \beta)]_{H P M}=|\tilde{Y}(1 ; r ; \beta)-\tilde{y}(1 ; r ; \beta)|
$$

where the fifth order HPM approximate solution in double parametric for fuzzy number for all $r \in[0,1]$ and $\beta \in$ $[0,1]$ is illustrated in Tables (1-6) and Figure 2 as follows:

Table 1. Fifth-order HPM solution of Eq. (13) at $x=1$ and $\beta=0$ for all $r \in[0,1]$

\begin{tabular}{|c|c|c|c|}
\hline $\boldsymbol{r}$ & $\underline{\boldsymbol{y}}(\mathbf{1} ; \boldsymbol{r})$ & $\underline{\boldsymbol{Y}(\mathbf{1}, \boldsymbol{r})}$ & $\underline{\mathbf{E}}(\mathbf{1}, \boldsymbol{r})$ \\
\hline 0 & 0 & 0 & 0 \\
\hline 0.2 & 0.5436299819942914 & 0.5436563656918091 & 0.000026383697517728955 \\
\hline 0.4 & 1.0872599639885827 & 1.0873127313836182 & 0.000079151092553075840 \\
\hline 0.6 & 1.6308899459828743 & 1.6309690970754274 & 0.000105534790070915820 \\
\hline 0.8 & 2.1745199279771654 & 2.1746254627672363 & 0.000131918487588311700 \\
\hline 1 & 2.7181499099714570 & 2.7182818284590450 & \\
\hline
\end{tabular}

Table 2. Fifth-order HPM solution of Eq. (13) at $t=1$ and $\beta=0.2$ for all $r \in[0,1]$

\begin{tabular}{|c|c|c|c|}
\hline $\boldsymbol{r}$ & $\underline{\boldsymbol{y}}(\mathbf{1} ; \boldsymbol{r})$ & $\underline{\boldsymbol{Y}}(\mathbf{1}, \boldsymbol{r})$ & $\underline{\boldsymbol{E}}(\mathbf{1}, \boldsymbol{r})$ \\
\hline 0 & 1.0872599639885827 & 1.0873127313836182 & 0.00005276739503545791 \\
\hline 0.2 & 1.4134379531851575 & 1.4135065507987037 & 0.00006859761354616190 \\
\hline 0.4 & 1.7396159423817323 & 1.7397003702137890 & 0.00008442783205664384 \\
\hline 0.6 & 2.0657939315783070 & 2.0658941896288745 & 0.00010025805056734782 \\
\hline 0.8 & 2.3919719207748820 & 2.3920880090439600 & 0.00011608826907805181 \\
\hline 1 & 2.7181499099714570 & 2.7182818284590450 & 0.00013191848758831170 \\
\hline
\end{tabular}

Table 3. Fifth-order HPM solution of Eq. (13) at $\mathrm{x}=1$ and $\beta=0.4$ for all $\mathrm{r} \in[0,1]$

\begin{tabular}{|c|c|c|c|}
\hline $\boldsymbol{r}$ & $\underline{\boldsymbol{y}}(\mathbf{1} ; \boldsymbol{r})$ & $\underline{\boldsymbol{Y}}(\mathbf{1}, \boldsymbol{r})$ & $\underline{\boldsymbol{E}}(\mathbf{1}, \boldsymbol{r})$ \\
\hline 0 & 2.1745199279771654 & 2.1746254627672363 & 0.00010553479007091582 \\
\hline 0.2 & 2.2832459243760237 & 2.2833567359055980 & 0.00011081152957448381 \\
\hline 0.4 & 2.3919719207748820 & 2.3920880090439600 & 0.00011608826907805181 \\
\hline 0.6 & 2.5006979171737402 & 2.5008192821823220 & 0.00012136500858161980 \\
\hline 0.8 & 2.6094239135725985 & 2.6095505553206833 & 0.00012664174808474370 \\
\hline 1 & 2.7181499099714570 & 2.7182818284590450 & 0.00013191848758831170 \\
\hline
\end{tabular}

Table 4. Fifth-order HPM solution of Eq. (13) at $\mathrm{x}=1$ and $\beta=0.6$ for all $\mathrm{r} \in[0,1]$

\begin{tabular}{|c|c|c|c|}
\hline $\boldsymbol{r}$ & $\overline{\boldsymbol{y}}(\mathbf{1} ; \boldsymbol{r})$ & $\overline{\boldsymbol{Y}}(\mathbf{1}, \boldsymbol{r})$ & $\overline{\boldsymbol{E}}(\mathbf{1}, \boldsymbol{r})$ \\
\hline 0 & 3.2617798919657480 & 3.261938194150854 & 0.00015830218510570 \\
\hline 0.2 & 3.1530538955668900 & 3.1532069210124930 & 0.00015302544560302 \\
\hline 0.4 & 3.0443278991680320 & 3.0444756478741306 & 0.00014774870609857 \\
\hline 0.6 & 2.9356019027691733 & 2.9357443747357688 & 0.00014247196659544 \\
\hline 0.8 & 2.8268759063703150 & 2.8270131015974070 & 0.00013719522709187 \\
\hline 1 & 2.7181499099714570 & 2.7182818284590450 & 0.00013191848758831170 \\
\hline
\end{tabular}


Table 5. Fifth-order HPM solution of Eq. (13) at $\mathrm{x}=1$ and $\beta=0.8$ for all $\mathrm{r} \in[0,1]$

\begin{tabular}{|c|c|c|c|}
\hline $\boldsymbol{r}$ & $\overline{\boldsymbol{y}}(\mathbf{1} ; \boldsymbol{r})$ & $\overline{\boldsymbol{Y}}(\mathbf{1}, \boldsymbol{r})$ & $\overline{\boldsymbol{E}}(\mathbf{1}, \boldsymbol{r})$ \\
\hline 0 & 4.349039855954331 & 4.3492509255344730 & 0.00021106958014183164 \\
\hline 0.2 & 4.022861866757756 & 4.0230571061193880 & 0.00019523936163157174 \\
\hline 0.4 & 3.696683877561181 & 3.6968632867043016 & 0.00017940914312042366 \\
\hline 0.6 & 3.370505888364607 & 3.3706694672892160 & 0.00016357892460927560 \\
\hline 0.8 & 3.044327899168032 & 3.0444756478741306 & 0.00014774870609857160 \\
\hline 1 & 2.718149909971457 & 2.7182818284590450 & 0.00013191848758831170 \\
\hline
\end{tabular}

Table 6. Fifth-order HPM solution of Eq. (13) at $\mathrm{x}=1$ and $\beta=1$ for all $\mathrm{r} \in[0,1]$

\begin{tabular}{|c|c|c|c|}
\hline $\boldsymbol{r}$ & $\overline{\boldsymbol{y}}(\mathbf{1} ; \boldsymbol{r})$ & $\overline{\boldsymbol{Y}}(\mathbf{1}, \boldsymbol{r})$ & $\overline{\boldsymbol{E}}(\mathbf{1}, \boldsymbol{r})$ \\
\hline 0 & 5.436299819942914 & 5.436563656918090 & 0.00026383697517662340 \\
\hline 0.2 & 4.8926698379486220 & 4.8929072912262810 & 0.00023745327765922752 \\
\hline 0.4 & 4.3490398559543310 & 4.3492509255344730 & 0.00021106958014183164 \\
\hline 0.6 & 3.8054098739600395 & 3.8055945598426630 & 0.00018468588262354757 \\
\hline 0.8 & 3.2617798919657480 & 3.2619381941508540 & 0.00015830218510570760 \\
\hline 1 & 2.7181499099714570 & 2.7182818284590450 & 0.00013191848758831170 \\
\hline
\end{tabular}

\section{HPM}

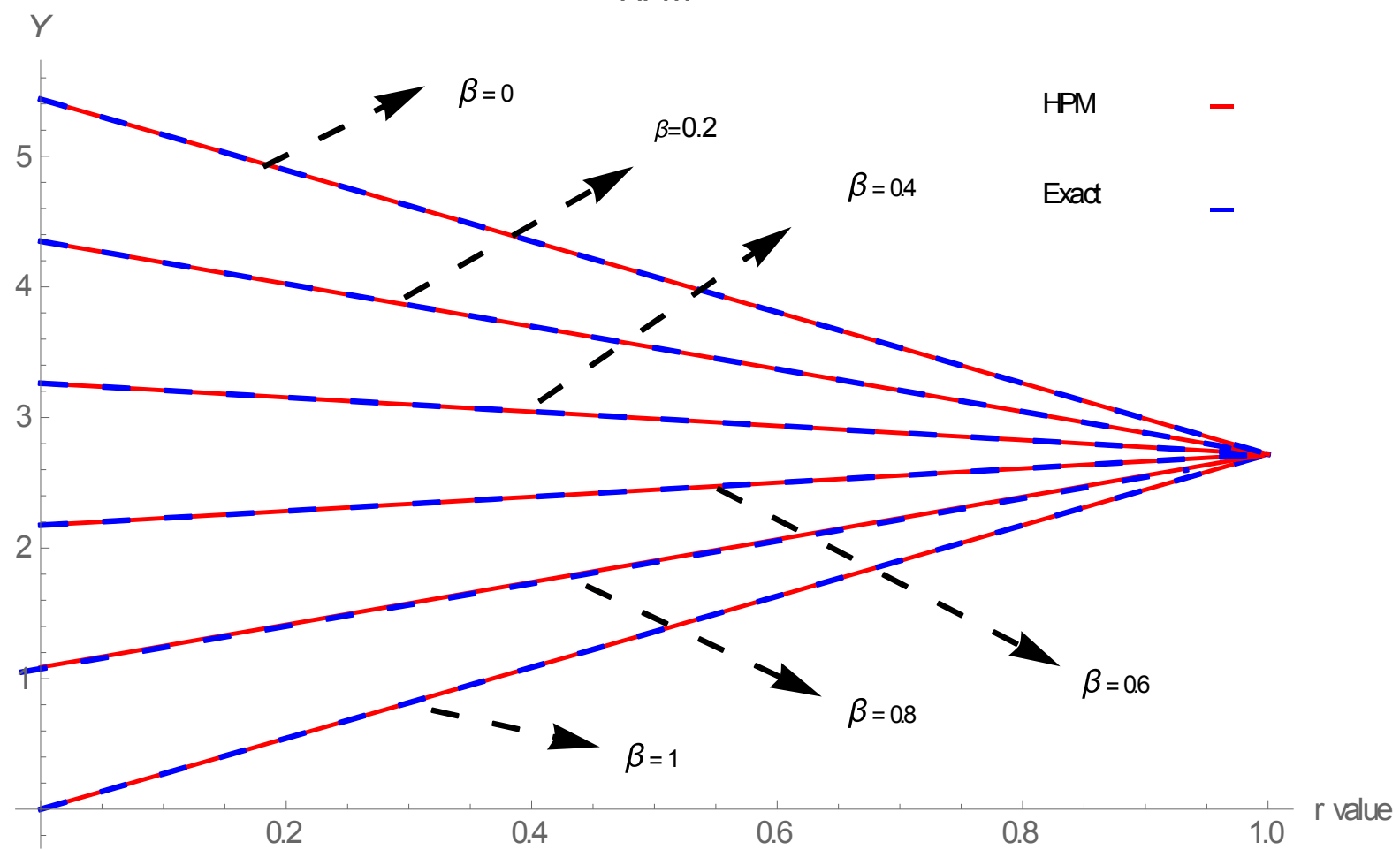

Figure 2. Comparison of fifth-order HPM and the exact solution at $x=1$ for different values of $\beta \in[0,1]$ and for all fuzzy level sets $r \in[0,1]$. 
From Tables 1 and 6, the lower and upper bound solution of Eq. (13) is obtained with fifth-order HPM solution when $\beta=0$ and 1 respectively that satisfies the fuzzy differential equations solution for all level sets $r \in[0,1]$ for $x=1$. Additionally, for each $\beta=0.2,0.4,0.6$ and 0.8 also follow the fuzzy differential equations solution for all level sets $r \in[0,1]$ for $x=1$ and other results can be obtained for all values of $x \in[0,1]$.

This analysis is illustrated in Figure 2 in the form of triangular fuzzy number, presenting and comparing the results under double parametric form fuzzy number of fifth order HPM and the exact solution of Eq. (13) for different values of $\beta \in[0,1]$.

\section{Conclusions}

A new form of HPM has successfully been constructed based on the approach of double parametric form of fuzzy numbers to solve first order DDEs using fuzzy-set theory properties. In this work, homotopy perturbation method (HPM) has successfully been analyzed, altered and applied to the approximate solution of first order FDDE using a double parametric form of fuzzy numbers. The proposed double parametric form approach is found to be easy and straightforward with less computational work. The performance of the method is shown using a triangular fuzzy number. It is interesting to note of the values of $\beta \in[0,1]$ satisfy the fuzzy differential equation solution in the form of the triangular fuzzy number of all fuzzy level set.

\section{Acknowledgments}

This research was supported by Ministry of Higher Education (MoHE) of Malaysia through Fundamental Research Grant Scheme (FRGS/1/2018/STG06/UUM/02/10).

\section{REFERENCES}

[1] A. Alomari, S. M. Noorani, and R. Nazar, Solution of delay differential equation by means of homotopy analysis method, Acta Applicandae Mathematicae, 108, 395-412, 2009.

[2] F. Salehi, M. A. Asadi, M. M. Hosseini, Solving system of DAEs by Modified Homotopy Perturbation Method, Journal of Computer Science \& Computational Mathematics, Vol. 22(6), 29-33, 2012.

[3] S. Vilu, R. Rozita Ahmad, and U. Khair Salma Din, Variational Iteration Method and Sumudu Transform for Solving Delay Differential Equation, International Journal of Differential Equations, 2019,1-6, 2019.

[4] Buckley, J., \& Feuring, T, Introduce to fuzzy partial differential equations, Fuzzy Sets and Systems, 105, 241-
248.1999.

[5] Omer, A and Omer, O., A Prey and Predator Model with Fuzzy Initial Values', Hacettepe Journal of Mathematics and Statistics, 41(2)pp 387-395, 2013.

[6] Tapaswini, S and Chakravery, S., Numerical Solution of Fuzzy Arbitrary Order Predator-Prey Equations', Applications and Applied Mathematics, 8(1), 647-673, 2013.

[7] El Naschie, M. S., From Experimental Quantum Optics to Quantum Gravity Via a Fuzzy Kahler Manifold', Chaos Solution and Fractals, 2 5, 969-977, 2005.

[8] Abbod, M. F., Von Keyserlingk, D. G., Linkens, D. A and Mahfouf, M. (2001) 'Survey of Utilization of Fuzzy Technology in Medicine and Healthcare', Fuzzy sets and system, Vol.120, pp. 331-349.

[9] S. Narayanamoorthy and T. L. Yookesh, Approximate Method for Solving the Linear Fuzzy Delay Differential Equations, Discrete Dynamics in Nature and Society Volume 2015, pp. 1-9, 2015.

[10] N. Mikaeilvand, and L. Hossieni, The Taylor Method for Numerical Solution of Fuzzy generalized Pantograph Equations with Linear Functional Argument, International Journal of Industrial Mathematics, 2(2), 115-127, 2010.

[11] J.-H. He, Homotopy perturbation technique, Computer Methods in Applied Mechanics and Engineering, 178(3-4), 257-262, 1999.

[12] J.-H. He, A coupling method of a homotopy technique and a perturbation technique for non-linear problems, nternational Journal of Non-Linear Mechanics, 35(1), 7-43, 2000.

[13] C. Chun, H. Jafari and Y.-I. Kim, Numerical method for the wave and non-linear diffusion equations with the homotopy perturbation method, Computers \& Mathematics with Applications, 57(7), 1226-1231, 2009.

[14] Sarmad A. Altaie, Ali F. Jameel, Azizan Saaban, Homotopy Perturbation Method Approximate Analytical Solution of Fuzzy Partial Differential Equation, IAENG International Journal of Applied Mathematics, 49(1), 22-28, 2019.

[15] J. Biazar, and H. Ghazvini, Convergence of the Homotopy Perturbation Method for Partial Differential equations, Nonlinear Analysis, 10(5), 2633-2640, 2009.

[16] Tapaswini, S., and Chakraverty, S. (2013), Numerical solution of uncertain beam equations using double parametric form of fuzzy numbers. Applied Computational Intelligence and Soft Computing, 2013, pp. 100-111.

[17] Dubois, D and Prade, H. Towards fuzzy differential calculus, Part 3: Differentiation', Fuzzy Sets and Systems, 8, pp. 225-233, 1982.

[18] Bodjanova, S., Median Alpha-Levels of A Fuzzy Number, Fuzzy Sets and Systems, 157(7), 879-891, 2006.

[19] Fard, O. S., An Iterative Scheme for the Solution of Generalized System of Linear Fuzzy Differential Equations', World Applied Sciences Journal, 7, 1597-11604.

[20] Diptiranjan. B and S. Chakraverty, New approach to solve fully fuzzy system of linear equations using single and 
double parametric form of fuzzy numbers, Indian Academy of Sciences, 40(1), 35-49, 2015.

[21] Guo, X., Shang, D and Lu, X. Fuzzy Approximate Solutions Of Second-Order Fuzzy Linear Boundary Value Problems', Journal of Boundary Value Problems, 2013, 1-17, 2013.

[22] Zadeh, L. A., Toward A Generalized Theory of Uncertainty, Information Sciences,. 172(2), 1-40, 2005.
[23] Smita, T and Chakraverty, S., Numerical Solution of Uncertain Beam Equations Using Double Parametric Form of Fuzzy Numbers, Applied Computational Intelligence and Soft Computing, 2013, 1-8,2013.

[24] Jameel, A F, Saaban, A., Ahadkulov, H., and Alipiah, F. M. Approximate solution fuzzy pantograph equation by using homotopy perturbation method, Journal of Physics: Conference Series, 980 (1). 2017. 